\title{
Surveying on MIMO Technology for Future Wireless Communication
}

\author{
Sandeep Kumar Kulkarni, Raju Yanamshetti
}

\begin{abstract}
Massive MIMO is an extension of traditional MIMO with the exception that the BSs in massive MIMO are equipped with large number of antennas, usually hundred or more. This large number of antennas provide several positive advantages towards wireless communication with respect to increasing volume of data traffic. Each antenna is capable of serving multiple users simultaneously leading to reduction in power consumption as well as data rate amplification. Additionally, narrow and more focused beams are pointed to individual user devices located at the cell edge thereby upgrading of downlink signal quality. Using massive MIMO technique also increases reliability of the links, reduces noise effects, and mitigates and interference. With increasing number of users gets service, the throughput of the system also increases.
\end{abstract}

Keywords: Massive MIMO, Base stations, 5G, MIMO-NOMA,

\section{INTRODUCTION}

As approximately two-thirds of world population will have access to Internet giving rise to nearly 5.3 billion of Internet users. In addition, most of the data usage will be for watching video. Traditional MIMO may exhibit limitations fulfilling such a high data rate demand but massive MIMO has the capability of fulfilling this high demand for wireless multimedia services in real time with 10 Gbps data rate [82]. That's why; the author provides a complete survey on all of the research directions todate regarding recent advancements, research directions, and future scenarios regarding massive MIMO.

\subsection{Overview of massive MIMO}

The basic idea behind massive MIMO is integrating high number of antennas at the base station for the purpose of providing simultaneous service to many live users operating within same time and frequency domain. Before proceeding further, there is a need of discussing about the difference between traditional MIMO and massive MIMO.

Manuscript received on October 05, 2021.

Revised Manuscript received on October 18, 2021.

Manuscript published on November 30, 2021.

* Correspondence Author

Sandeepkumar Kulkarni*, Department of Electronics and Instrumentation, Gulbarga University, Gulbarga (Karnataka), India.

Dr. Raju Yanamshetti Kulkarni, Department of Electronics and Communication Engineering, Gulbarga University, Gulbarga (Karnataka), India.

(C) The Authors. Published by Blue Eyes Intelligence Engineering and Sciences Publication (BEIESP). This is an open access article under the CC BY-NC-ND license (http://creativecommons.org/licenses/by-nc-nd/4.0/) stated in CISCO annual report (2018-2023) [1], by 2023,

In general, with respect to number of users being serviced, MIMO can be classified into two categories: single-user MIMO (SU-MIMO) and multi-user MIMO (MU-MIMO) as depicted in figure 1 below.

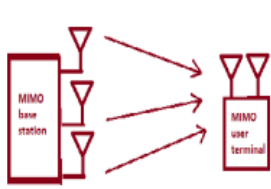

a)

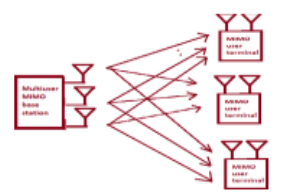

b)
Figure 1: a) Single user MIMO and b) Multi-user MIMO In case of SU-MIMO, both the transmitter and the receiver provisioned with multiple antennas but the drawback is that only one user is served at a time. In this case, one of the transmitter/receiver can be a BS. Performance enhancing schemes like beamforming, space-time coding, and multiplexing of several data streams with respect to space can't be employed to their full extent and a trade-off scheme should be followed. On the other hand, MU- MIMO uses antenna array at the BS for serving several user devices with single antenna. All the active users get a share of the space divided wireless channel and without using the techniques of joint encoding and detection, the users are capable of transmitting and receiving data. The BS connection to the users is simultaneous by exploiting different spatial signatures at the base station caused by different users. Though MUMIMO is advantageous over SU-MIMO, one important challenge in MU-MIMO is the occurrence of interference among same channel users which can be managed by using complex algorithms at the receiving device for proper decoding of bits in the message. In addition, MU-MIMO requires highly explicit beamforming and beforehand allocation of resources to the user terminals.

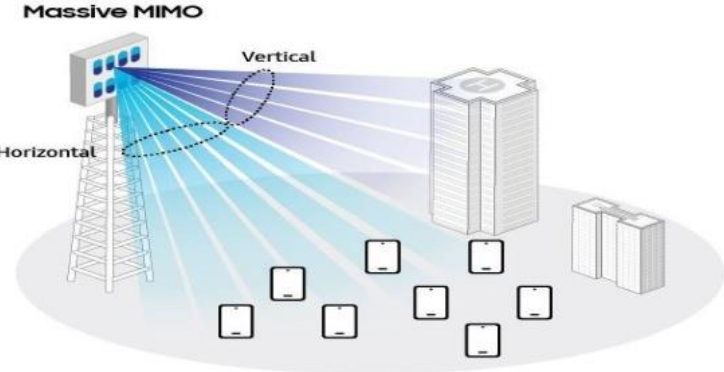

Figure 2: Massive MIMO beamforming [3]

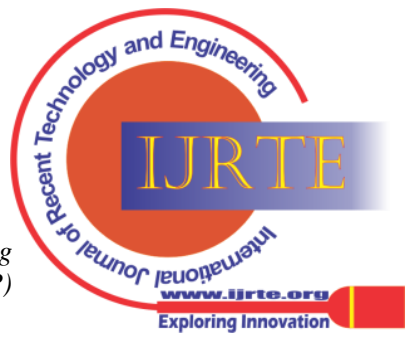


Basically, massive MIMO is the extension of classical MIMO along with the integration of two innovative techniques: MUMIMO and 3D beamforming. The concept of beamforming refers to the technique of finding most effective data transmission path for a particular user device. Most of the current cellular networks handle their beam radiations in a horizontal manner, which is referred to as 2D beamforming. Unlike 2D beamforming, 3D beamforming supports more users by generating elevation and azimuth plane radiation patterns. In short, massive MIMO can be thought as a 3D beamforming technique enhancing horizontal as well as vertical coverage.

\section{CHALLENGES OF MASSIVE MIMO}

Irrespective of having a large number of benefits of using massive MIMO, there are a few fundamental problems which are need to be solved. According to literature the challenges to overcome in massive MIMO are mentioned as more or less the factors mentioned below.

- Channel approximation

- Pilot symbol impurity

- Identification of signals

- Multi-stream beam forming

- Finding optimal set of users

- Energy effectiveness

Primary real world implementation challenges of massive MIMO have been discussed below along with several research works carried out between 2010 to 2021 for mitigation of these challenges.

\subsection{Channel Estimation with Time Division Duplexing (TDD) vs. frequency Division Duplexing (FDD)}

In case of FDD scheme, the absence of channel reciprocation property brings overhead in obtaining CSI. For downlink transmission, the BS transmits $\mathrm{N}$ orthogonal pilots to the users. From the received pilot symbol, each user approximates the CSI and sends their estimates back to the BS through uplink transmission. For uplink signaling, users send their orthogonal pilot symbols to the BS and aiding these received pilots, the BS approximates the CSI.

As there is a large overhead in using FDD in massive MIMO CSI estimation, most of the researchers have considered using of TDD. The primary objective behind using TDD is to bypass the overhead of downlink channel estimation as the resource amount necessary to estimate is directly proportional to the large number of antennas in the BS. As a result, deploying of massive MIMO is a challenging task and drawing researchers' attention in a great manner. With increase in BS antennas, transmission time of downlink pilot increases and consequently FDD channel approximation scheme fails. This event can be mitigated by the aid of TDD channel estimation scheme which is armed with channel reciprocation property.

\subsection{Pilot Contamination}

This drawback is related to CSI estimation. For massive MIMO TDD, channel approximation is carried out during uplink orthogonal pilot transmission from each user. These received pilots help the BS to approximate the uplink as well as downlink channel. But, as the time duration for predicting average signal phase and bandwidth is limited, the pilot length and the available orthogonal codes for a specific codebook is also limited. In this situation, two or more users may need to use the same pilot. If the users are in two adjacent cells, then channel estimation is corrupted by interference between users in the adjacent cell and istermed as pilot contamination.

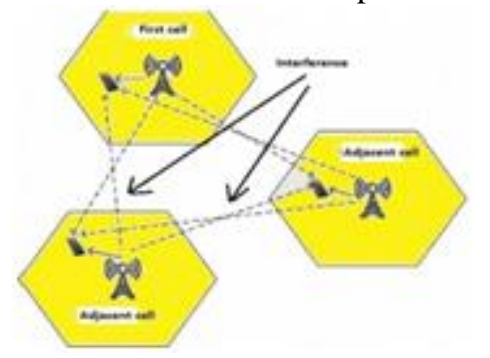

Figure 3: Pilot contamination between adjacent cells

Researchers have tried various options in reducing the effect of pilot contamination. So it can be concluded that although plenty of research works have been performed on pilot decontamination in massive MIMO, the solutions have not been completely accepted to be applied for commercial purpose. Hence still there are scopes of research in this domain.

\subsection{Identification of individual transmitted signals:}

The massive MIMO BS contains a high number of antennas to make them capable of serving a high number of user devices having one or few antennas in the same frequency range. So the BS needs to separately identify multiple data transmission from the users. The uplink transmission can interfere or overlap with each other making it difficult for the BS to correctly decode user transmission. The BS has to identify the most possible transmitted data bits from each individual transmission depending on the CSI and the received transmissions. So, the BS must take help of a reliable detector. As depicted in the Figure 4 below, the BS has several antennas and serving many user devices with one antenna. The signal transmission from the user devices may progress through different paths and ultimately converging at the BS resulting in overlapping of transmissions. This is the reason behind wide research for designing an optimal signal identifier.

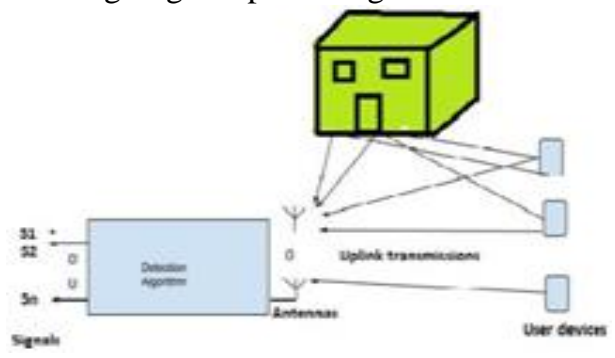

Figure 4: Uplink signal detection in massive MIMO

Published By:

Blue Eyes Intelligence Engineering and Sciences Publication (BEIESP)

(C) Copyright: All rights reserved.

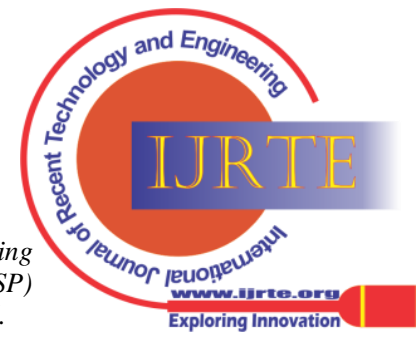


Massive MIMO signal identification systems which are less complex, employs repetitive techniques, such as, Neumann Series Approximation (NSA), Richardson's technique, Repetitive Over-Relaxation (ROR) technique, and Jacobi's repetitive method. But the problem is that amount of complexity in computation is not reduced much with respect to traditional linear decoding. Researchers have also tried with several other linear decoding such as least square regression selection, approximate message passing (AMP), conjugate gradient etc. But, unfortunately, no method has proved itself optimal for signal decoding in massive MIMO uplink transmission.

\subsection{Multi-stream beamforming:}

In massive MIMO, the concept of precoding refers to focusing the transmission beam with more than one stream for transmission to more than one user. The CSI is approximated from the uplink pilot sequences or from the feedbacks conveyed by the user terminals. The problem is that the approximated CSI may not be accurate and the inaccuracy is introduced by environmental influences on the wireless channel. So, accurate downlink transmission depends on approximated CSI and the precoding technique employed. In general, the precoding technique refers to weighting or successively encoding the signal to be transmitted downlink. For example, in linear precoding (or beamforming), beams are generated by weighing the phase and amplitude of the antennas for pointing energy to an individual user. But the advantages of precoding technique for massive MIMO system are somehow mitigated by introduction of complex computations with increasing number of antennas. That's why massive MIMO system should go for less complex and practically efficient precoders.

\subsection{Finding optimal set of users:}

The BSs of massive MIMO server large number of users by using large number of antennas. As a result of this concurrent communication, the event of multi-user interference occurs and the system throughput is degraded. At the time of downlink transmission, use of precoding techniques can minimize multiuser interference. But, there can be a situation when number of user devices to be served can be much more than the number of antennas in the BS as antennas in the BSs are constant in number. In these situations, appropriate user scheduling techniques are to be used before applying precoding technique. For achieving outstanding bandwidth efficiency, massive MIMO depends on orthogonality of user channels. But, in case of line-of-sight (LOS) communication, identical channel vectors of some users being served concurrently can degrade the throughput performance of the overall system. Hence, user devices having identical vectors should be identified and served in isolated time slots and providing maximum possible data rates. This technique can provide unbiased service to all users as well as optimizing bandwidth efficiency. There are several attempts for finding an optimal scheduling algorithm for enhancing sum capacity. But, most of the past algorithms were unable to minimize the computational complexity in case of large number of antennas. Recent works have focused on more improved algorithms for an optimal solution.

\subsection{Energy effectiveness:}

Massive MIMO has the capability of achieving a great amount of energy efficacy by acquiring high bandwidth effectiveness with low consumption of power. To be in the scene with stability, massive MIMO should function by consuming less energy but still achieving desired data capacity limit. Researchers should consider about a significant design factor here known as bit/joule Energy Efficacy (EE) which can be stated as

$$
E E=R / P
$$

Here, $\mathrm{R}$ denotes system throughput and $\mathrm{P}$ denotes energy consumed to achieve R.

Now, the point is that as the number of antennas grows, the bandwidth efficacy also increases, as more power will be consumed with more number of antennas as well as more number of users.

Hence, the objective is that the amount of power consumed should be kept under a certain level irrespective of number of antennas and/or users increase. Depending on this fact, researchers have attempted to design less complex and economic channel approximation, precoding, detection and user scheduling algorithms to maintain a low level in power consumption. Design of antennas as well as power amplifiers have also been investigated for minimizing power consumption.

\subsection{Hardware imperfections:}

One topic that has not been explored much by the researchers is to design low-cost hardware for massive MIMO BS irrespective of degrading the system performance. High number of antennas in massive MIMO take part in minimizing the factors of interference, noise, and fading. But the problem is that employment of large number of antennas is the reason of more complex system as well as more cost. The objective is to minimize computational complexity and hardware volume for deploying a massive MIMO system. So, the way is to use low-cost hardware. But, low-cost hardware components exhibit several impurities like amplifier distortion, phase noise, magnetization noise etc. As a result, the overall system performance is affected. In addition, baseband hardware and data converters consume more power with more number of antennas. In reality, hardware imperfections can't be removed in its entirety. So, there is a need to design appropriate algorithm for reducing the effect of hardware imperfections. There are various attempts to avoid this problem; but most of them are successful with limited number of antennas, not with large number of antennas. One solution can be using of exclusive hardware running in concurrent manner.
Published By:

Blue Eyes Intelligence Engineering and Sciences Publication (BEIESP) (C) Copyright: All rights reserved.

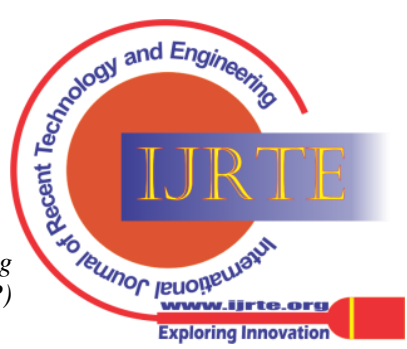




\section{Surveying on MIMO Technology for Future Wireless Communication}

A research work has demonstrated that a low Peak to Average Power Ratio (PAPR) can be used to minimize the effect of imperfections due to low-cost amplifier at the transmitter side [16].

\subsection{Role of Machine learning in massive MIMO network:}

Recently, the concept of Machine Learning/Deep Learning are very popular and handy for several human-made intelligent applications such as processing of natural language, network security, and autonomous systems. Even the learning algorithms are being used extensively in designing 5G/6G networks. This tremendous popularity of the learning algorithms has made the researchers explore their applicability in massive MIMO system also. Due to dynamic behavior of the learning algorithms, they can be very much helpful in optimizing highly complex and high power-consuming massive MIMO system. Important research issues in massive MIMO like channel approximation, signal recognition, load balancing etc. can be implemented using learning algorithms.

\subsection{Research considerations for massive MIMO 5 G and ahead communication:}

Although several research works are being carried out in massive MIMO, still it has not been deployed in practical. To make it deployed for practical use, more extensive research has to be carried out on above mentioned factors. In addition, exponential increase in number of mobile devices has made the research world think about the scenarios beyond 5G that has resulted in research on very new technologies like millimeter waves, terahertz, visible light communication, spatial modulation, cell-free massive MIMO, MIMO based routing algorithms etc. Probable domains and directions of research on massive MIMO for 5G and future communications have been discussed below.

\subsubsection{Millimeter-Wave communication (mmWave communication).}

Usually, the wireless cellular communication uses the frequency less than $6 \mathrm{GHz}$. Frequencies greater than $6 \mathrm{GHz}$ are used by applications like remote sensing, amateur radio, terahertz computation etc. In recent years, there is a huge increase in data traffic volume which has resulted in overcrowding of radio wave spectrum leading to poor service to users. To mitigate this drawback, one way is to use frequencies greater than $6 \mathrm{GHz}$ for radio communication and frequency range $30-300 \mathrm{GHz}$ has been taken into consideration, known as millimeter wave (mmWave). Currently, a great deal of research work is being carried out on this domain. Various research topics on mmWave massive MIMO have been considered by the researchers. Detailed survey works on mmWave and mmWave with massive MIMO communication have been carried out in [17][18]. As conventional digital precoding methods impose inflated cost and power consumption, combination of analog and digital precoding has been investigated by the researchers [18] [19]. Channel approximation in mmWave massive MIMO can be a problem due to pilot contamination and several mitigation attempts have been made [20] [21]. mmWave also has some drawbacks like inability of passing through obstacles and smaller wavelength.

\subsubsection{Cell-free massive MIMO}

The techniques like smart cities and Internet of Everything can overwhelm cellular networks due to a huge volume of nonhuman generated data traffic in addition to human generated data traffic. So, in future, we may need to go cell-free. In case of cell-free (CF) massive MIMO, a great number of autonomous antennas are scattered over a large area for proving concurrent service to handful of user devices.

The property of easy signal processing and unbiased service to all users have made it a potential candidate for future wireless communication. An elaborated survey has discussed about channel approximation, signal recognition, power management, precoding techniques, access point selection in CF massive MIMO along with future research orientations [22].

\subsubsection{Massive-MIMO-NOMA}

The concept of non-orthogonal multiple access in 5G has the capability to serve many users by using same resources of time and frequency. If NOMA is fused with massive MIMO in a single unit, there is a high possibility of bandwidth efficiency (i.e. spectral efficiency, SE) and energy efficiency (EE) optimization. An investigative work on massive-MIMONOMA has presented mathematical expressions for SignalInference-to-Noise-Ratio (SINR), BER, outage probability and capacity [23]. The author has also demonstrated the improvement in SE and EE. In another work, a scheme for securing privileged information in massive-MIMO-NOMA has been designed [24].

\subsubsection{Routing algorithms with MIMO support}

In traditional or massive MIMO, efficient resource allocation techniques can only be complemented by optimized routing path selection. For selecting an optimal routing path over various media, corresponding interference status of the media must be taken into account. In addition, allocation status of the frequency channel decides the corresponding radio channel selected. As a consequence, cross-layer methods must be chosen with the objective of maximized performance of massive MIMO network. A comparative exploration of current MIMO-based routing algorithms has been presented in [25]. In another work, the author has designed an innovative routing algorithm for applying in massive MIMO heterogeneous network and demonstrated its dominance over present techniques

\section{CONCLUSION}

A survey on massive MIMO communication has been presented.

- To properly estimate the signal transmitted, the receiver must know about the channel state between the transmitter and the receiver.

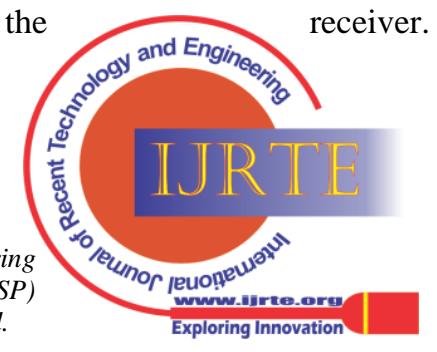


The channel state is actually a combination of various properties of the channel along with the effect of scattering, fading on the channel. For a successful data transmission, CSI must be estimated beforehand. So, an accurate, less complex channel approximation algorithm should be designed and implemented.

- For the purpose of approximating the channel at the BS, the user devices transmit orthogonal pilot sequences during uplink transmission which are used by the BS to obtain CSI. The problem is that only a handful of orthogonal pilot sequences are available leading to reuse of pilot symbols. This event gives rise to the problem of pilot contamination and poses a great challenge on massive MIMO communication.

- $\quad$ CSI received at BS has impurities and during downlink transmission, the BS uses an approximation of the CSI. The downlink transmission quality greatly depends on this predicted CSI and that's why a suitable precoding technique should be employed for minimization of interference and bandwidth efficiency.

- $\quad$ Power expenditure in massive MIMO increases with the increase in number of antennas and the users. So, the goal of the researchers should be designing economical algorithms leading to power consumption minimization.

- Identifying overlapped user signals from uplink transmission at the BS is a challenging and widely explored problem in the literature. But, a suitable signal identification algorithm is yet to be designed as existing algorithms are unable to minimize computational complexity with increasing number of antennas.

- $\quad$ For decreasing massive MIMO deployment cost, less complex and small-sized hardware should be designed.

- $\quad$ Researchers should find out how mm Wave frequency can be suitably integrated with massive MIMO to meet huge data traffic demand in the future.

- Combination of massive MIMO and NOMA should be explored for extensively for optimal use of nonorthogonal multiple access scheme in 5G and later.

- A great deal of success of massive MIMO depends on a suitably designed routing path with reduced noise and interference. Hence researchers should be attentive in this direction. From the above discussion it can be concluded that although massive MIMO system provides us with several advantages suitable for next generation wireless communication, still more research should be conducted to make this system suitable for deployment in reality.

\section{REFERENCES}

1. Cisco Annual Internet Report (2018-2023), White paper, Cisco public, 2020

2. Erik G. Larsson et al, "Massive MIMO for Next Generation Wireless Systems", IEEE Communications Magazine, Vol. 52, Issue 2, pp. 186195, 2014

3. "Massive MIMO for New Radio", Technical White Paper, Samsung, 2020

4. Hien Quoc Ngo et al, "Blind Estimation of Effective Downlink Channel Gains in Massive MIMO",Iternational Conference on Acoustics, Speech and Signal Processing (ICASSP), 2015

5. Chao-Kai Wen et al, "Channel Estimation for Massive MIMO Using Gaussian-Mixture Bayesian Learning”, IEEE t transactions on Wireless Communications, Vol. 14, Issue 3, pp. 1356-1368, 2015

6. Hongxiang Xie et al, "A Simple DFT-aided Spatial Basis Expansion Model and Channel Estimation Strategy for TDD/FDD massive MIMO Systems", arXiv 2015, arXiv:1511.048412016, 2016

7. Chao-Kai Wen et al, "Bayes-Optimal Joint Channel-and-Data Estimation for Massive MIMO with Low-Precision ADCs", IEEE Transactions on Signal Processing, Vol. 64, Issue 10, pp. 1-17, 2015

8. De Mi et al, "A Novel Antenna Selection Scheme for Spatially Correlated Massive MIMO Uplinks with Imperfect Channel Estimation", arXiv 2014, arXiv:1403.2902, 2014

9. Gao, $\mathrm{Z}$ et al, "Structured compressive sensing-based spatio-temporal joint channel estimation for FDD massive MIMO", IEEE Trans. Communication, Vol. 64, pp. 601-617, 2016

10. $\mathrm{Xu}, \mathrm{W}$ et al, "Downlink Performance of Massive-MIMO Systems Using EVD-Based Channel Estimation", IEEE Trans. Vehicular Technology, Vol. 66, Issue 4, pp. 3045-3058, 2017

11. Huang, W et al, "Beam-blocked Channel Estimation for FDD Massive MIMO with Compressed Feedback", IEEE Access 2017, doi:10.1109/ACCESS.2017.2715984

12. Imran Khan et al, "A Robust Channel Estimation Scheme for 5G Massive MIMO Systems", Wireless Communications and Mobile Computing, Vol. 2019

13. Felipe A. et al, "Channel estimation for massive MIMO TDD systems assuming pilot contamination and flat fading", IEEE Access, Vol. 5, pp. 17733 - 17741, 2017

14. [14] Chang-Jae Chun et al, "Deep Learning Based Channel Estimation for Massive MIMO Systems", IEEE Wireless Communications Letters, Vol. 8, Issue 4, pp. 1228 - 1231, 2019

15. Bule Sun et al, "Interference Cancellation Based Channel Estimation for Massive MIMO Systems with Time Shifted Pilots", IEEE Transactions on Wireless Communications, Vol. 19, Issue 10, pp. 6826 - 6843, 2020

16. [16] Ture Peken et al, "Blind channel estimation for massive MIMO", Analog Integrated Circuits and Signal Processing, Vol. 91, Issue 2, 2017

17. Hien Quoe Ngo et al, "EVD-Based Channel Estimation in Multicell Multiuser MIMO Systems with Very Large Antenna Arrays", IEEE International Conference on Acoustics, Speech and Signal Processing (ICASSP), 2012

18. Ralf R. Muller et al, "Blind Pilot Decontamination", IEEE Journal of Selected Topics in Signal Processing, Vol. 8, Issue 5, pp. 773 - 786, 2014

19. Md. Abdul Latif Sarker et al, "A Fast Channel Estimation and The Reduction of Pilot Contamination Problem for Massive MIMO Based On a Diagonal Jacket Matrix", 4th International Workshop on Fiber Optics in Access Network (FOAN), 2013

20. David Neumann et al, "Suppression of Pilot-Contamination in Massive MIMO Systems", IEEE 15th International Workshop on Signal Processing Advances in Wireless Communications (SPAWC), 2014 [R21] Wan A. W. M. Mahyiddin et al, "Pilot Contamination Reduction Using Time-Shifted Pilots in Finite Massive MIMO Systems, IEEE 80th Vehicular Technology Conference (VTC2014-Fall), 2014

21. Zhangkai Luo et al, "Pilot Contamination Mitigation via a Novel TimeShift Pilot Scheme in Large-Scale Multicell Multiuser MIMO Systems", International Journal of Antennas and Propagation, 2016

22. Shi Jin et al, "On Massive MIMO Zero-Forcing Transceiver Using TimeShifted Pilots", IEEE Transactions on Vehicular Technology, Vol. 65, Issue 1, pp. J59 - 74, 2015

23. Te Wei et al, "Optimized Time-Shifted Pilots for Maritime Massive MIMO Communication Systems", 26th Wireless and Optical Communication Conference (WOCC), 2017

24. Vidit Saxena et al, "Mitigating Pilot Contamination by Pilot Reuse and Power Control Schemes for Massive MIMO Systems", IEEE 81st Vehicular Technology Conference (VTC Spring), 2015

25. Tedros Salih et al, "Pilot Reuse Factor with Large Scale Fading Precoding for Massive MIMO", arXiv:1702.02562v1 cs.IT], 2017

26. Jiancun Fan et al, "Pilot contamination mitigation by fractional pilot reuse with threshold optimization in massive MIMO systems", Digital Signal Processing, Vol. 78, pp. 197-204, 2018

\section{Published By:}

Blue Eyes Intelligence Engineering and Sciences Publication (BEIESP)

(c) Copyright: All rights reserved.

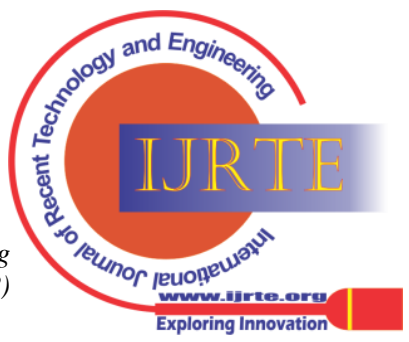




\section{Surveying on MIMO Technology for Future Wireless Communication}

27. Jesper H. Sørensen et al, "Uncoordinated pilot decontamination in massive MIMO systems", EURASIP Journal on Wireless Communications and Networking, pp. 1-15, 2017

28. Ahmed Hesham Mehana et al, "Pilot hopping algorithm for massive multiple-input multiple-output", IET Communications, Vol. 12, Issue 4, pp. 393-401, 2018

29. Nazanin Kalantarinejad et al, "Joint distance-based user grouping and pilot assignment

30. schemes for pilot decontamination in massive MIMO systems", The International Journal of Communication Systems, pp. 1-17, 2019

31. Muhammad Irshad Zahoor et al, "Pilot Decontamination Using AsynchronousFractional Pilot Scheduling in Massive MIMO Systems", Sensors, Vol. 20, Issue 21, pp. 1-21, 2020

32. [32] Xinhua Nie et al, "Joint Pilot Allocation and Pilot Sequence Optimization in Massive MIMO Systems",IEEE Access, Vol. 8, pp. 60637 - 60644, 2020

33. Yasaman Omid et al, "A New Pilot Assignment Scheme for Pilot Decontamination of Multi-Cell Massive MIMO Systems", 27th Iranian Conference on Electrical Engineering (ICEE), 2019

34. Xiliang Luo et al, "IRS-Based TDD Reciprocity Breaking for Pilot Decontamination in Massive MIMO", IEEE Wireless Communications Letters (Revised), Vol. 10, Issue 1, pp. 102 - 106, 2021

35. Cheng Hu et al, "Pilot Decontamination in Multi-Cell Massive MIMO Systems via Combining Semi- Blind Channel Estimation with Pilot Assignment”, IEEE Access, Vol. 8, pp. 152952 - 152962, 202

\section{AUTHOR'S PICTURE AND BIBLIOGRAPHY:}

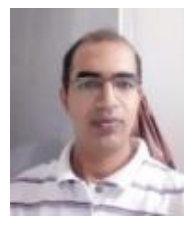

Sandeepkumar Kulkarni received his Bachelor Degree in Electronics and Instrumentation from Gulbarga University, Gulbarga and Master Degree in Power Electronics from VTU -Belgaum in 2001 and 2006 respectively. In 2013, he joined in VTU-Belgaum as Ph.D part time student in MIMO microstrip antenna group and carrying his research work under the direction of Dr. Raju Yanamshetti, PDACE-Gulbarga. He has Membership of IEI, KRVP and KSP. Guided several UG and PG students and presented papers in National, International and UGC approved journals.

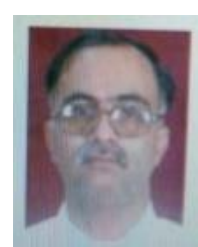

Dr. Raju Yanamshetti Kulkarni received his Bachelor Degree in Electronics and Communication Engineering from Gulbarga University, Gulbarga and Master Degree in Power Electronics from Gulbarga University, Gulbarga and Ph.D in Electrical Engineering from Jadhavpur University in 1986, 1991 and 2012 respectively. He has Membership of ISTE, ISOI, and IETE. Presented several papers under National and International journals. Guiding several research scholars under various areas. Area of research in VLSI Design, real time embedded systems and solid state power controllers.

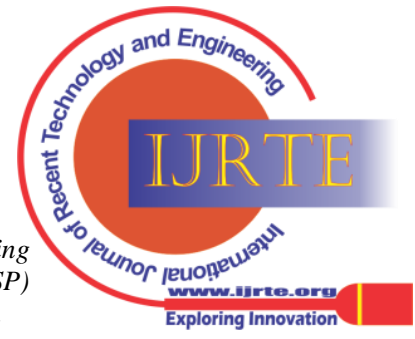

